\title{
Run-time Evolution for Embedded Component-Oriented Systems
}

\author{
Yves Vandewoude \\ Department of Computer Science \\ KULeuven \\ Celestijnenlaan 200A \\ B-3001 Leuven, Belgium \\ email: Yves.Vandewoude@cs.kuleuven.ac.be
}

\author{
Yolande Berbers \\ Department of Computer Science \\ KULeuven \\ Celestijnenlaan 200A \\ B-3001 Leuven, Belgium \\ email: Yolande.Berbers@cs.kuleuven.ac.be
}

\begin{abstract}
This position paper describes ongoing work in which the Java-based SEESCOA component system is extended with functionality for run-time evolution. First, an assessment is made of the state-of-the-art in dynamic updating, and the applicability of existing systems for dynamic updating is examined. Then a new approach is presented, in which the concept of ports is used to redirect messages between components. The problem of class-file reloading in the JVM is avoided by modification of the classes at load-time to include version information. The predictability requirement of embedded systems is assured by updating all component instances at once.
\end{abstract}

\section{KEY WORDS}

Dynamic Updating, Embedded Systems, Component Systems

\section{Introduction}

Run-time evolution is defined as the ability to change part of the system's functionality or structure during its execution. Changing a program without stopping it opens up a wide range of opportunities such as fixing bugs, adding functionality, software distribution to name a few. In addition, live updates can lead to significant performance enhancements through run-time optimization.

Since run-time change is far from trivial to accomplish, designers traditionally sought alternatives to circumvent the need for it: regularly scheduled downtimes and redundancy are frequently used. However, in many cases redundant hardware is not present since they add to cost and complexity.

In particular, embedded devices (characterized by their long life cycle) often require, and can always benefit from seamless upgrading of their software. Dynamic software evolution would allow the system to adapt its functionality in response to frequent changes in the device's context.

Although embedded systems have a history of low level design in which reuse and maintainability often are sacrificed for speed and efficiency, research on component- oriented development of embedded software is a hot topic. For software engineers, component-oriented development integrates the object-oriented mechanisms for composition and reuse with other features as concurrency, persistence and distribution. Users benefit from tailor made functionality through adaptation of ready-made components. Perhaps the intention of Nokia to release 100 million Javaenabled units by the end of 2003 ([14]) illustrates best that in the near future embedded applications are not necessarily low-level applications. After all, the significantly reduced time-to-market and development costs, fit extremely well in the context of large-scale distributed systems and are excellently suited for mass deployment.

However, genuine reuse requires a formal definition of a component. In the context of $\mathrm{SEESCOA}^{1}$, a cooperation project between the KULeuven and other Belgian universities, a component architecture was developed for embedded systems. One of the merits of SEESCOA is establishing a formal definition of a component and introducing an approach on the connection of different components ([15]). In addition, a tool to support component-oriented design (the CCOM tool: [16]) and a run-time environment were developed. A final objective of the project is to extend the component-architecture to support dynamic reconfiguration.

In the context of run-time evolution, a distinction is made between functional and structural changes. Whereas functional changes include the addition of new code to a running system in order to modify its functionality, structural changes refer to reconfigurations that change the relationship between different components of the system, or replicate portions of the application for execution on a different machine.

Further distinctions are made between programmed modifications (foreseen during design-time) and unanticipated changes (discovered after the deployment of the system). For the SEESCOA component system, we are mainly interested in functional and unanticipated changes.

\footnotetext{
${ }^{1}$ SEESCOA stands for Software Engineering for Embedded Systems using a Component-Oriented Approach. The project is funded by the Belgian IWT.
} 


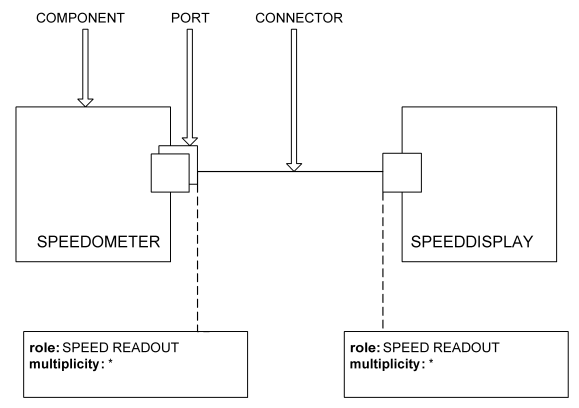

Figure 1: Component, Port and Connector

This article is further organized as follows: in section 2, a short introduction on the SEESCOA component architecture is given. Then we examine the different mechanisms for run-time evolution (section 3) and their suitability for component-oriented embedded systems. In section 4 we describe ongoing work in adjusting the SEESCOA runtime environment. A conclusion is given in section 5.

\section{The SEESCOA Component- Architecture}

In SEESCOA a component is defined as a runtime object offering coherent behaviour. It communicates with other components through asynchronously sent messages. In order to send messages, every component has a number of ports. In a formal XML based description of a port, a specification of the port protocol is given. The description of components is defined at four levels:

Syntactic Level: Description of the message signatures.

Semantic Level: Description of pre- and post conditions.

Synchronization Level: Description of the sequence of messages, loops and alternative paths. This level is described by means of a timed MSC ${ }^{2}$.

QoS Level: The component's resource needs and behaviour.

Ports with a compatible protocol can be connected using a connector. This is illustrated in figure 1.

The SEESCOA run-time system implements intercomponent communication through message delivery. Messages sent out or received by a component can be seen, filtered or rerouted using message handlers. For details on the design of the component architecture, we refer the interested reader to [17]. The SEESCOA runtime is implemented in Java, and deployed on an embedded device running the Kaffe Virtual Machine.

\footnotetext{
${ }^{2}$ Message Sequence Chart
}

\section{Assessment of systems for run-time configuration}

A lot of work in the field of dynamic updates has been done in the past and has resulted in a variety of different systems. However, they all have a different approach, and emphasize different aspects of the huge pool of often conflicting requirements. To give a complete overview is beyond the scope of this paper, and we refer to $[6,8,13]$ for excellent surveys. Instead, based on the classification described in [18], our discussion will be focused on the applicability of different systems in component-oriented development for embedded systems.

Many systems focus on updating procedural systems (e.g. $[4,6,8])$. Using some form of indirection, a method-call is diverted to a new version of the procedure. Wrappers are used to handle interface changes and in some cases state is transferred using mapper functions. Updates can be initiated by the application itself or by an external shell. This way, updates can be delayed until real-time constraints can be met. However, applying procedure-based systems to object-oriented or component-oriented applications is extremely difficult, since this breaks $\mathrm{OO}$ abstractions and type safety. Therefore, these systems will not be considered any further.

A straightforward method for implementing dynamic reconfiguration in an object-oriented system is by using the Strategy Design Pattern ([5]). This simple solution allows for changing an algorithm or strategy at run-time, but it requires that the application is designed with future changes in mind, and that all possible strategies must be known at compile-time. Therefore, it is not eligible for unanticipated evolution.

With Conus, Kramer and Magee pioneered in the field of component-oriented evolution. In [10] they describe a system in which a programmer can specify the desired changes in a declarative way. The concept of quiescent nodes is used for safe removal of a component from the running system. Since they did not attempt to transfer state between different versions of a component, their system needs extension in order to truly implement dynamic updating.

Using provisions available in the Chorus Operating system, Hauptmann and Wasel achieve deterministic timing behaviour during updates ([7]). Just like the SEESCOA component system, it uses the concept of ports to reroute messages between objects. Their system however, is very hard to implement on other operating systems.

A very flexible approach can be found in metaarchitectures (e.g. [2, 12]). Through reification of object oriented concepts (class, method-call, ...), a meta-model is build on top of the application. By changing this metamodel, structural changes of the application are possible. A major advantage is that this provides a clean separation between the application code (base-code) and the code respon- 
sible for the reconfiguration (meta-code). In some cases ${ }^{3}$ it is possible to build an adaptation framework that is orthogonal to the application domain ([3]). However, the large overhead resulting from this approach is not acceptable in an embedded context.

Other systems focus specifically on dynamic Java. In [11], Malabarba et al. focus on being full consistency with the Java language and its type safety. They extended the default Java class loader in a way that classdefinitions can be replaced and objects or dependent classes can be updated. The replacement is initiated by the user by explicit calls to the class loader in the application program.

With Gilgul, Costanza proposed an extension to the Java language that supports Dynamic Object Replacement ([1]). In order to achieve this, Gilgul introduces some indirections. Nevertheless, the overhead is never very large, and occurs only during object replacements. An implementation of Gilgul is available for the Kaffe Virtual Machine.

An interesting approach to implement dynamic reconfiguration is Kniesel's work on dynamic delegation with Lava (a variation of Java) ([9]). It allows for interface changes and does not require the application to be developed with evolution in mind. The concept of delegation is very well suited for component-oriented systems, since it allows child references to its parent to change dynamically. With delegation however, all versions of a component remain in memory leading to a significant overhead that is not acceptable for embedded systems.

All the above systems provide us with concepts, either useful to support runtime reconfiguration, or relevant to component-oriented embedded systems and they will be used in our own approach.

\section{Ongoing work}

In the SEESCOA component system, messages between components are sent to ports. The destination port is located by the component runtime using a lookup table. When a port receives a message, it will pass on the information to its associated component. This concept of ports allows us to rewire components at runtime and therefore simplifies dynamic reconfiguration. By moving a port from one component to another, all pending and future messages are rerouted. Therefore, replacing a component with a new version should not cause any difficulties in inter-component communications.

However, the components and the runtime are implemented in Java. This results in two problems:

Intra-component communications: A component typically consists of multiple classes. Those classes com-

\footnotetext{
${ }^{3}$ Some patterns of adaptation might require application specific knowledge. Therefore, not all adaptation are possible while retaining orthogonality with the application domain.
}

municate with each other using ordinary method calls, and do not pass through the component system.

Loading Classes: When a new version of a component is loaded, it is likely that an already instantiated class must be replaced with a new version. This is not possible using default Java class loaders, since reloading of a class is not possible.

When an entire component is updated at once, intracomponent communications are not an issue. However, considering that our target platform is an embedded system and that the duration of an update is related to the size and complexity of the change, partial updating of a component can be useful. This can be achieved by rewriting our system in Gilgul .

The class loading problem will be tackled using classfile modification. The class-files will be modified at loadtime by changing their name to contain version information. Since there is no direct inter-component communication with Java calls, only classes within the same component can call each other directly. Therefore, all calling classes are known at load time, and a simple renaming procedure will suffice.

Statetransfer between components is a last step in the process of dynamic updates. Initially, a new component version should implement a copy-constructor: the new version is responsible to adopt the state of its predecessor. Although straightforward, there are some drawbacks to this approach.

- An additional burden is placed on the designer of the component to implement this copy constructor.

- The component should leave its state public or must provide an interface in order for future versions to read it.

The next phase of the project will focus on automating this process. A tool will be created to generate (portions of) the transition code between two versions, based on semantic information provided by the component designer. The Java Native Interface can be used to access private state information. With this tool, a separation of the application code and the transition code will be guaranteed.

One problem remains: when will the components be updated? An often used method for updating objects is updating on demand: objects are updated when they are first referenced. This method avoids the need for an expensive updating phase. A drawback of this method is that the duration of accessing a component is no longer predictable. Hence, an alternative approach is desired for embedded systems. One possibility is to update all instances of a component at once. Although time consuming, the update itself can be scheduled like any other task in the system. A second alternative is to have new component instances use the new version, but not to update existing instances. We decided to implement the first alternative, since the second prevents 
many useful program restructurings and might cause problems considering the long life cycle of an embedded device.

\section{Conclusion}

After an introduction to the SEESCOA component system, an assessment was made on the state-of-the art in dynamic updating. Our focus was on the applicability to componentoriented embedded systems. Using this experience, we then proposed an approach to add support for runtime evolution to the component system. Ports are used to reroute messages between components. Reloading classes is avoided by including version information in the class name at load-time using bytecode transformer. Updating portions of a components is made possible using the Gilgul extension to the Java programming language. To assure that no unpredictabilities are introduced in the updated system, all components are updated at once. In the first version, state will be achieved by requiring components to implement a copy-constructor. A more structured approach with tool support is planned in the near future.

\section{References}

[1] Costanza, P. Dynamic object replacement and implementation-only classes. In Proceedings of the 6th International Workshop on Component-Oriented Programming at ECOOP (Budapest, Hungary, June 2001).

[2] Dowling, J., And Cahill, V. Dynamic software evolution and the k-component model. In Workshop on Software Evolution, OOPSLA (2001).

[3] Dowling, J., Schaefer, T., Cahill, V., HARASZTI, P., AND REDMOND, B. Using reflection to support dynamic adaptation of system software: A case study driven evaluation. In OOPSLA Workshop on Object-Oriented Reflection and Software Engineering (Denver, Colorado, November 1999), pp. 169188.

[4] Frieder, O., And Segal, M. E. On dynamically updating a computer program: From concept to prototype. The Journal of Systems Software 14, 2 (February 1991), 111-128.

[5] Gamma, E., Helm, R., Johnson, R., And VlisSIDES, J. Design Patterns: Elements of Reusable Object-Oriented Software. Addison Wesley, Massachusetts, 1994.

[6] Gupta, D. On-line Software Version Change. PhD thesis, Department of Computer Science and Engineering, Indian Institute of Technology, Kanpur, Nobember 1994.
[7] Hauptmann, S., And Wasel, J. On-line maintenance with on-the-fly software replacement. In Proceedings of the 3th International Conference of Configurable Distributed Systems (1996), IEEE Computer Society Press.

[8] Hicks, M. Dynamic Software Updating. PhD thesis, Department of Computer and Information Science, University of Pennsylvania, June 2001.

[9] KNIESEL, G. Type-safe delegation for run-time component adaptation. In Proceedings of ECOOP 99 (Lisbon, Portugal, June 1999).

[10] Kramer, J., And Magee, J. The evolving philosophers problem: Dynamic change management. IEEE Transactions on Software Engineering 16, 11 (1990), 1293-1306.

[11] Malabarba, S., Pandey, R., Gragg, J., Barr, E., AND BARNES, J. F. Runtime support for type-safe dynamic java classes. In Proceedings of the 14th European Conference on Object-Oriented Programming (June 2000).

[12] Redmond, B., And CAhill, V. Iguana/j: Towards a dynamic and efficient reflective architecture for java. In Workshop on Reflection and Meta-Level Architectures at 14th European Conference on ObjectOriented Programming (Cannes, France, June 2000).

[13] Segal, M. E., ANd Frieder, O. On-the-fly program modification: Systems for a dynamic updating. IEEE Software 10, 2 (1993), 53-65.

[14] Suutarinen, J. Java in mobile world. Sun Developers Connection - J2ME \& Wireless Java, Februari 2002.

[15] Urting, D., Baelen, S. V., Holvoet, T., And BERBERS, Y. Embedded software development: Components and contracts. In Proceedings of the IASTED International Conference Parallel and Distributed Computing and Systems (2001), pp. 685-690.

[16] Urting, D., Baelen, S. V., Holvoet, T., Rigole, P., VAndewoude, Y., And Berbers, Y. A tool for component based design of embedded software. In Proceedings of Tools Pacific 2002 (Februari 2002).

[17] VAn Belle, W. Refinement of the component architecture. SEESCOA Deliverable, October 2001.

[18] Vandewoude, Y., And Berbers, Y. An overview and assessment of dynamic update methods for component-oriented embedded systems. In Proceedings of The International Conference on Software Engineering Research and Practice (Las Vegas, USA, June 2002). 